\title{
Distributed Interference Management in Two-Tier CDMA Femtocell Networks
}

\author{
Duy Trong Ngo, Student Member, IEEE, Long Bao Le, Member, IEEE, Tho Le-Ngoc, Fellow, IEEE, \\ Ekram Hossain, Senior Member, IEEE, and Dong In Kim, Senior Member, IEEE
}

\begin{abstract}
This paper proposes distributed joint power and admission control algorithms for the management of interference in two-tier femtocell networks, where the newly-deployed femtocell users (FUEs) share the same frequency band with the existing macrocell users (MUEs) using code-division multiple access (CDMA). As the owner of the licensed radio spectrum, the MUEs possess strictly higher access priority over the FUEs; thus, their quality-of-service $(\mathrm{QoS})$ performance, expressed in terms of the prescribed minimum signal-to-interference-plusnoise ratio (SINR), must be maintained at all times. For the lower-tier FUEs, we explicitly consider two different design objectives, namely, throughput-power tradeoff optimization and soft QoS provisioning. With an effective dynamic pricing scheme combined with admission control to indirectly manage the cross-tier interference, the proposed schemes lend themselves to distributed algorithms that mainly require local information to offer maximized net utility of individual users. The approach employed in this work is particularly attractive, especially in view of practical implementation under the limited backhaul network capacity available for femtocells. It is shown that the proposed algorithms robustly support all the prioritized MUEs with guaranteed QoS requirements whenever feasible, while allowing the FUEs to optimally exploit the remaining network capacity. The convergence of the developed solutions is rigorously analyzed, and extensive numerical results are presented to illustrate their potential advantages.
\end{abstract}

Index Terms-Femtocell, macrocell, CDMA, power control, admission control, QoS protection, distributed interference management.

\section{INTRODUCTION}

$\mathbf{F}$ EMTOCELLS have recently emerged as a promising technology to increase wireless network capacity, extend

Manuscript received January 12, 2011; revised August 19, 2011; accepted January 1, 2012. The associate editor coordinating the review of this paper and approving it for publication was A. Chockalingam.

This work was supported in part by the Natural Science and Engineering Research Council of Canada (NSERC) Grants, the Alexander Graham Bell Canada Graduate Scholarship, the McGill Engineering Doctoral Award, and the MKE, Korea, under the ITRC support program (NIPA-2011-(C10901111-0005)). Part of this paper was presented at the 2011 IEEE Vehicular Technology Conference (VTC-Fall), San Francisco, CA, USA.

D. T. Ngo and T. Le-Ngoc are with the Department of Electrical and Computer Engineering, McGill University, Montréal, QC, Canada H3A 2A7 (e-mail: duy.ngo@mail.mcgill.ca; tho.le-ngoc@mcgill.ca).

L. B. Le, (corresponding author) is with the Centre Énergie Matériaux Télécommunications, Institut National de la Recherche Scientifique (INRSEMT), Université du Québec, Montréal, QC, Canada H5A 1K6 (e-mail: long.le@emt.inrs.ca).

E. Hossain is with the Department of Electrical and Computer Engineering, University of Manitoba, Winnipeg, MB, Canada R3T 5V6 (e-mail: ekram@ee.umanitoba.ca).

D. I. Kim is with the School of Information and Communication Engineering, Sungkyunkwan University, Suwon, Korea 440-746 (e-mail: dikim@ skku.ac.kr).

Digital Object Identifier 10.1109/TWC.2012.012712.110073 cellular coverage and introduce new services [1], [2]. Femtocell solution offers significant economic benefits compared to the traditional cell-partitioning approach for which a large number of expensive base stations (BSs) are typically required. By deploying low-cost femtocell (home) BSs, indoor users can enjoy high-speed wireless communication due to the close proximity between themselves and their own home BSs. In this new wireless solution, limited signaling data can be transmitted over the backhaul networks via residential wireline broadband access links, e.g., digital subscriber lines (DSL), without any further investment.

Since femtocells operate in the licensed spectrum owned by the macrocell network, it is imperative to limit the crosstier interference from the femtocell users (FUEs) to the macrocell [3]. One of the central research topics is to develop autonomous interference management schemes such that (i) the quality-of-service (QoS) requirements of the existing macrocell users (MUEs) with higher access priority are always maintained, and (ii) the residual network capacity is effectively exploited by the newly-deployed FUEs so as to optimize their own performance. This is the case for two-tier networks based on either code-division multiple access (CDMA) or orthogonal frequency-division multiple access (OFDMA) [4], [5].

The development and implementation of distributed interference management solutions for femtocell networks are challenging for two main reasons [6]-[10]. First, given that the wireline network infrastructure (e.g., DSL links) may only provide limited capacity for the exchange of signaling information, it is rather difficult to centrally coordinate femtocell and macrocell BSs to perform such management. Second, because of the different access tariffs applicable for these two types of users, the MUEs have strictly higher priority over the FUEs in accessing the underlying radio spectrum.

The literature on distributed power control in traditional CDMA wireless networks is rich. One of the most popular solutions is the one presented in [11], proven to converge to a Pareto-optimal solution whenever the minimum signal-tointerference-plus-noise ratios (SINRs) of all the users can be supported. In the case of infeasible SINR targets, admissioncontrol or user-removal algorithms are introduced in [12], [13]. The works in [14]-[17] investigate several other power control schemes from a game-theoretical point of view. In most instances, the devised distributed algorithms converge to the Nash equilibrium of the corresponding power-control games. In addition, various pricing schemes are developed to achieve a balance between maximizing the total network utility and minimizing the power consumption, and/or to improve the efficiency of the equilibrium solutions [18]-[21]. For 
homogeneous data-service multicell systems, [22] considers the distributed Pareto-optimal joint optimization of SINR assignment and power control. In the context of femtocell networks, [23]-[26] study various beamforming techniques to mitigate the undue cross-tier interference. Joint admission control and power management has also been examined in [27] for cognitive-CDMA networks.

In this work, we present joint power and admission control solutions for distributed interference management in two-tier CDMA-based femtocell networks. The fundamental difference between the setting considered in this paper and that investigated in traditional CDMA wireless networks is the differentiated classes of users with distinct access priorities and design requirements. The prioritized MUEs demand that their QoS requirements be always maintained in the first place, whereas the lower-tier FUEs attempt to optimize their performance by exploiting the remaining available system resource. Specifically, we investigate the following two practical scenarios: (i) the FUEs desire to balance between their achieved throughput and the corresponding power expenditure, and (ii) the FUEs demand certain "soft" QoS requirements, expressed in terms of the minimum attained SINRs. In lightlyloaded networks, we also propose an effective mechanism to better utilize the network capacity and thereby improve the performance of the MUEs. Convergence properties of the proposed algorithms are rigorously analyzed and potential extensions presented to further emphasize the attractiveness of the developed solutions.

It is noteworthy that, whilst closest in spirit with [28], our work distinguishes itself in, at least, two key aspects. Firstly, in representing the net utility of the FUEs, the study in [28] uses a penalty function that depends on the actual cross-tier interference, and thus requires explicit information about the cross-channel gains. It is, indeed, quite challenging to estimate these values due to the random fluctuations caused by shadowing and short-term fading. On the contrary, this paper proposes an effective dynamic pricing scheme combined with admission control to indirectly manage the cross-tier interference. Together with their distributive nature, the developed schemes are more tractable in view of practical implementation under the limited backhaul network capacity available for femtocells. Secondly, the choice of utility function for the MUEs in [28] does not always guarantee the minimum required SINRs to be achieved for these prioritized users. Instead, the joint power and admission control algorithms devised here, through the selection of a sigmoid function to represent the macrocell utility, are capable of robustly protecting the performance of all the active MUEs.

The rest of this paper is organized as follows: Section II introduces the system model under consideration and summarizes the assumptions applicable throughout the work. In Section III, distributed interference-management algorithms are proposed and the corresponding analysis is presented. Section IV discusses several practical issues regarding the implementation of the devised schemes, together with some possible extensions. Section V demonstrates the performance of the developed schemes by numerical results. Finally, Section VI concludes the paper.

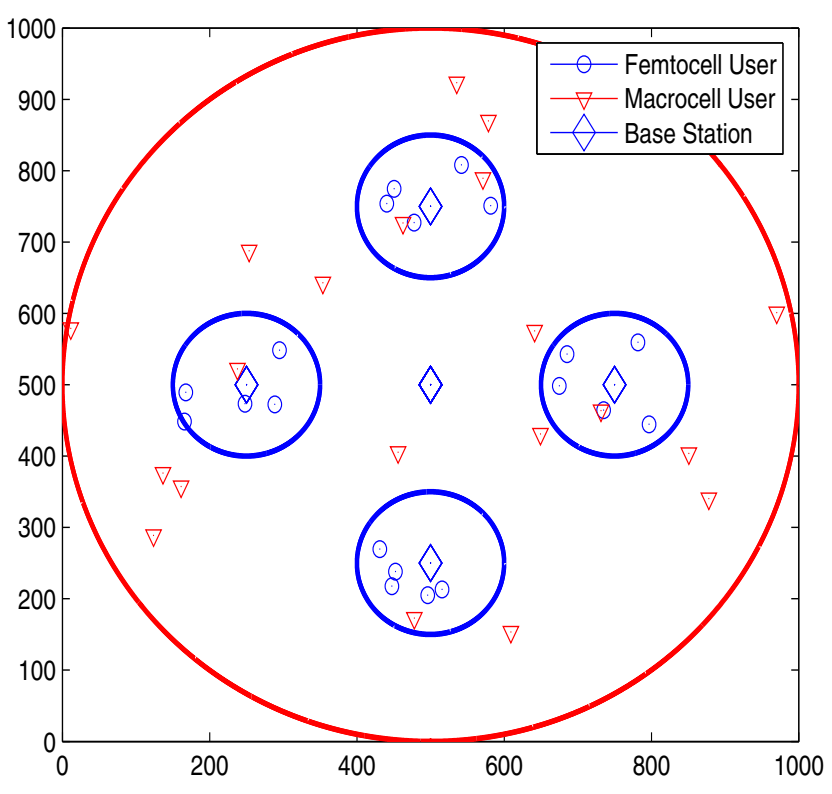

Fig. 1. Example of network topology and user placement in a two-tier network.

\section{System Model And Assumptions}

Consider a two-tier wireless network with power-controlled users. Specifically, we investigate the scenario where a macrocell serving $M$ macrocell users (MUEs) is underlaid with $K$ femtocells using code-division multiple access (CDMA). Assume that femtocell $i$ has $N_{i}$ users and define $N=\sum_{i=1}^{K} N_{i}$. We further assume that the association of the femtocell users (FUEs) with their closest femtocell base stations (BSs) is fixed during the runtime of the underlying power and admission control processes. Denote the set of all users by $\mathcal{L}$, and the set of MUEs and FUEs by $\mathcal{L}_{m}$ and $\mathcal{L}_{f}$, respectively. An example of such a network is illustrated in Fig. 1.

The results obtained in this work are applicable to both downlink and uplink scenarios. By "the transmitter of user $i$ " we refer to the BS that serves wireless terminal $i \in \mathcal{L}$ in the downlink case, whereas in the uplink case it is the wireless terminal $i$ itself. We consider a snapshot model where the channel gains are assumed to remain unchanged during the runtime of the power and admission control algorithms. Let $p_{i}$ be the transmit power of user $i$ and $\sigma_{i}$ the power of additive white Gaussian noise measured in the spectrum bandwidth at the receiving end of user $i \in \mathcal{L}$. Also, denote the channel gain from the transmitter of user $i$ to its receiver by $g_{i i}^{\prime}$, and that from the transmitter of user $j$ to the receiver of user $i \neq j$ by $g_{i j}$. Then, the received SINR of user $i \in \mathcal{L}$ can be written as

$$
\gamma_{i}=\frac{G g_{i i}^{\prime} p_{i}}{\sum_{j \neq i} g_{i j} p_{j}+\sigma_{i}}
$$

where $G$ is the processing gain of the system.

Note that the first term in the denominator of (1) includes both in-cell and cross-tier interferences, i.e., aggregated interference from all MUEs and FUEs except the considered user $i$ (which can be either a MUE or a FUE). In the downlink case, the channel gain $g_{i j}$ simply reduces to $g_{i i}^{\prime}$ for the incell interference, while $g_{i j}$ is termed the cross-channel gain for the cross-tier interference. For notational convenience, let 
$g_{i i}=G g_{i i}^{\prime}$ where the processing gain $G$ is absorbed into the channel gain $g_{i i}^{\prime}$. The received SINR of user $i \in \mathcal{L}$ can then be expressed as

$$
\gamma_{i}=\frac{g_{i i} p_{i}}{\sum_{j \neq i} g_{i j} p_{j}+\sigma_{i}} .
$$

In cellular wireless networks such as IS-95, WCDMA and LTE networks, regardless of the traffic types, a minimum SINR is required at the receiver for a minimum data rate to be supported. While the maintenance of such minimum SINR targets is well-justified for voice users to achieve a certain desired bit error rate (BER), it is also applicable to data users, especially those with delay-sensitive applications. In our modeling framework, different SINR thresholds are assigned to different users, depending on their access priority and application requirements. Given a desired threshold $\Gamma_{i}$, we assume that the prioritized MUE $i \in \mathcal{L}_{m}$ requires that

$$
\gamma_{i} \geq \Gamma_{i}
$$

On the other hand, each FUE $i \in \mathcal{L}_{f}$, which is of a lower access priority, is assumed to suppress transmission whenever its attained SINR falls below a predefined threshold $\gamma_{i}^{(f)}$. The rationale behind this assumption is that a negligible level of SINR would not help anything at all, but only create unnecessary interference to other users. Therefore, an active FUE $i \in \mathcal{L}_{f}$ must have that

$$
\gamma_{i} \geq \underline{\gamma}_{i}^{(f)}
$$

In this paper, we employ a utility function $U_{i}\left(\gamma_{i}\right)$ and a cost function $C_{i}\left(p_{i}\right)$ to represent the degree of satisfaction of user $i \in \mathcal{L}$ to the service quality and the cost incurred, respectively. It is the interest of user $i \in \mathcal{L}$ to maximize its own net utility, defined as

$$
U_{\mathrm{tot}, i}=U_{i}\left(\gamma_{i}\right)-C_{i}\left(p_{i}\right) .
$$

In fact, (5) is a standard way to define the payoff function for network entities (i.e., wireless users and BSs). Given the transmit power of other users, the net utility can be maximized by dynamic power adaptation performed at individual links.

Assume that $U_{i}\left(\gamma_{i}\right)$ is a strictly concave function with respect to $\gamma_{i}$, whereas $C\left(p_{i}\right)$ is convex in $p_{i}$. The necessary condition for the optimization of (5) can be obtained by taking the derivative of $U_{\text {tot }, i}$, which is also strictly concave in $p_{i}$, and equating to zero as follows.

$$
\frac{\mathrm{d} U_{\text {tot }, i}}{\mathrm{~d} p_{i}}=\frac{\mathrm{d} U_{i}}{\mathrm{~d} \gamma_{i}} \frac{\mathrm{d} \gamma_{i}}{\mathrm{~d} p_{i}}-\frac{\mathrm{d} C_{i}}{\mathrm{~d} p_{i}}=0 .
$$

Upon noting that $\mathrm{d} \gamma_{i} / \mathrm{d} p_{i}=g_{i i} / I_{i}=\gamma_{i} / p_{i}$, we have

$$
U_{i}^{\prime}\left(\gamma_{i}\right)=\frac{p_{i}}{\gamma_{i}} C_{i}^{\prime}\left(p_{i}\right)=\frac{I_{i}}{g_{i i}} C_{i}^{\prime}\left(p_{i}\right),
$$

where $I_{i}=\sum_{j \neq i} g_{i j} p_{j}+\sigma_{i}$ is the total noise and interference power at the receiving side of user $i \in \mathcal{L}$. From (7), the optimal target SINR can be derived as

$$
\widehat{\gamma}_{i}=f_{i}^{-1}\left(\frac{I_{i}}{g_{i i}} C_{i}^{\prime}\left(p_{i}\right)\right),
$$

where $f_{i}\left(\gamma_{i}\right)=U_{i}^{\prime}\left(\gamma_{i}\right)$. Based on $\widehat{\gamma}_{i}$ in (8), the following iterative power-update rule can be applied [20]:

$$
p_{i}(t+1)=\widehat{\gamma}_{i}(t) \frac{I_{i}(t)}{g_{i i}(t)}=\frac{\widehat{\gamma}_{i}(t)}{\gamma_{i}(t)} p_{i}(t),
$$

where $\gamma_{i}(t)$ is the actual SINR of user $i$ at iteration $t$. In fact, (9) represents a more general power-control rule compared with the following well-known power update (see, e.g., [11], [29]):

$$
p_{i}(t+1)=\frac{\Gamma_{i}}{\gamma_{i}(t)} p_{i}(t) .
$$

Specifically, the minimum required SINR $\Gamma_{i}$ on the right-hand side of (10) is replaced by the adaptive SINR threshold $\widehat{\gamma}_{i}(t)$ in (8).

In what follows, we will show how to choose appropriate functions $U_{i}\left(\gamma_{i}\right)$ and $C_{i}\left(p_{i}\right)$, together with their operating parameters, to design efficient distributed power and admission control algorithms for both MUEs and FUEs. The key aspect that makes the existing algorithms (such as those in [12], [13]) unsuitable for our current purpose is that the minimum SINRs of the prioritized MUEs should be maintained at all times. Accordingly, the FUEs must have their transmit powers properly controlled or, if needed, may even be removed for the sake of protecting the MUEs.

\section{Distributed Joint Power AND AdMission CONTROL AlgOrithms FOR TWO-TIER Networks}

\section{A. QoS Guarantees for Macrocell Users}

In the design of their power control scheme, [20] recommends the use of a sigmoid utility function and a linear cost function. For our problem at hand, by employing similar utility and cost functions for the MUEs and via properly tuning their control parameters, we can develop an efficient and robust power control algorithm that is capable of maintaining the minimum SINR requirements for these users. Specifically, we select the following utility and cost functions for MUE $i \in \mathcal{L}_{m}$ :

$$
\begin{aligned}
U_{i}\left(\gamma_{i}\right) & =\frac{1}{1+\exp \left[-b_{i}\left(\gamma_{i}-c_{i}\right)\right]}, \\
C_{i}\left(p_{i}\right) & =a_{i}^{(m)} p_{i} .
\end{aligned}
$$

Here, $b_{i}$ and $c_{i}$, respectively, control the steepness and the center of the sigmoid function, whereas $a_{i}^{(m)}$ is the pricing coefficient.

Function $U_{i}\left(\gamma_{i}\right)$ in (11) naturally captures the value of the service offered to user $i$. Noting that $U_{i}(0)=0, U_{i}(\infty)=1$, and that $U_{i}\left(\gamma_{i}\right)$ is increasing with respect to $\gamma_{i}$, it is clear that user $i$ is more and more satisfied with the offered service as the quality, expressed in terms of the achieved SINR $\gamma_{i}$, improves. On the other hand, power is itself a valuable system resource. The linear cost in (12) is chosen to reflect the expenses of power consumption to the user, while still retaining the simplicity of subsequent analysis. As will be shown later, the use of dynamic values of $a_{i}^{(m)}$ may significantly affect the resulting equilibrium of the developed algorithms.

Importantly enough, the choice of sigmoid function allows for the design of efficient schemes that guarantee the minimum 
SINRs imposed by the MUEs. Using (11) and (12), equation (7) can be rewritten as:

$$
U_{i}^{\prime}\left(\gamma_{i}\right)=f_{i}\left(\gamma_{i}\right)=\frac{a_{i}^{(m)} I_{i}}{g_{i i}} .
$$

From this relationship, it is straightforward to see that the optimal SINR target is

$$
\widehat{\gamma}_{i}=f_{i}^{-1}\left(\frac{a_{i}^{(m)} I_{i}}{g_{i i}}\right) .
$$

With the utility function defined in (11), an analytical form of (14) can be obtained as [20]:

$$
\widehat{\gamma}_{i}=c_{i}-\frac{1}{b_{i}} \ln \left[\frac{b_{i} g_{i i}}{2 a_{i}^{(m)} I_{i}}-1-\sqrt{\left(1-\frac{b_{i} g_{i i}}{2 a_{i}^{(m)} I_{i}}\right)^{2}-1}\right]
$$

Now, the line that goes through the origin and is tangent to the utility curve $U_{i}\left(\gamma_{i}\right)$ can be expressed as $U_{i}\left(\gamma_{i}\right)=$ $U_{i}^{\prime}\left(\gamma_{i}\right) \gamma_{i}$. At the tangent point $\gamma_{i, u}$, it is true that

$$
U_{i}\left(\gamma_{i, u}\right)=U_{i}^{\prime}\left(\gamma_{i, u}\right) \gamma_{i, u} .
$$

Since the cost function in (12) can also be rewritten as $C_{i}\left(p_{i}\right)=\left(a_{i}^{(m)} I_{i} / g_{i i}\right) \gamma_{i}$, it is required that $a_{i}^{(m)} I_{i} / g_{i i} \leq$ $U_{i}^{\prime}\left(\gamma_{i, u}\right)$ for a nonnegative total utility. On the other hand, the necessary and sufficient condition for $\widehat{\gamma}_{i}$ in (15) to achieve $U_{\text {tot }, i} \geq 0$ is $\widehat{\gamma}_{i} \geq \gamma_{i, u}$; otherwise, MUE $i$ simply suppresses its transmission and still gains zero total payoff. Therefore, by setting

$$
\gamma_{i, u}=\Gamma_{i},
$$

we can ensure that any active MUE (i.e., whose transmit power is strictly positive) will attain its minimum SINR target. In other words, an active MUE $i$ will eventually achieve SINR $\widehat{\gamma}_{i} \geq \gamma_{i, u}=\Gamma_{i}$ under this design. Some manipulations of (16) and (17) give [20]

$$
c_{i}=\Gamma_{i}-\frac{\ln \left(b_{i} \Gamma_{i}-1\right)}{b_{i}} .
$$

Upon substituting this value of $c_{i}$ to (15), we finally arrive at

$$
\begin{array}{r}
\widehat{\gamma}_{i}=\Gamma_{i}-\frac{\ln \left(b_{i} \Gamma_{i}-1\right)}{b_{i}}-\frac{1}{b_{i}} \ln \left[\frac{b_{i} g_{i i}}{2 a_{i}^{(m)} I_{i}}-1\right. \\
-\sqrt{\left(1-\frac{b_{i} g_{i i}}{2 a_{i}^{(m)} I_{i}}\right)^{2}-1} .
\end{array}
$$

In Fig. 2, we show the operating range of an active MUE $i$. With a sufficiently large $b_{i}$, function $U_{i}^{\prime}(\cdot)$ becomes very steep; therefore, the resulting $\gamma_{i}$ of user $i$ will be very close to its SINR threshold $\Gamma_{i}$. Also clear from Fig. 2 is that if the minimum required SINRs of all the MUEs are feasible, we can make them all active by setting $a_{i}^{(m)}$ sufficiently small. Specifically, given its total received interference and noise power $I_{i}$, MUE $i \in \mathcal{L}_{m}$ is active if

$$
a_{i}^{(m)}<g_{i i} U_{i}^{\prime}\left(\Gamma_{i}\right) / I_{i} .
$$

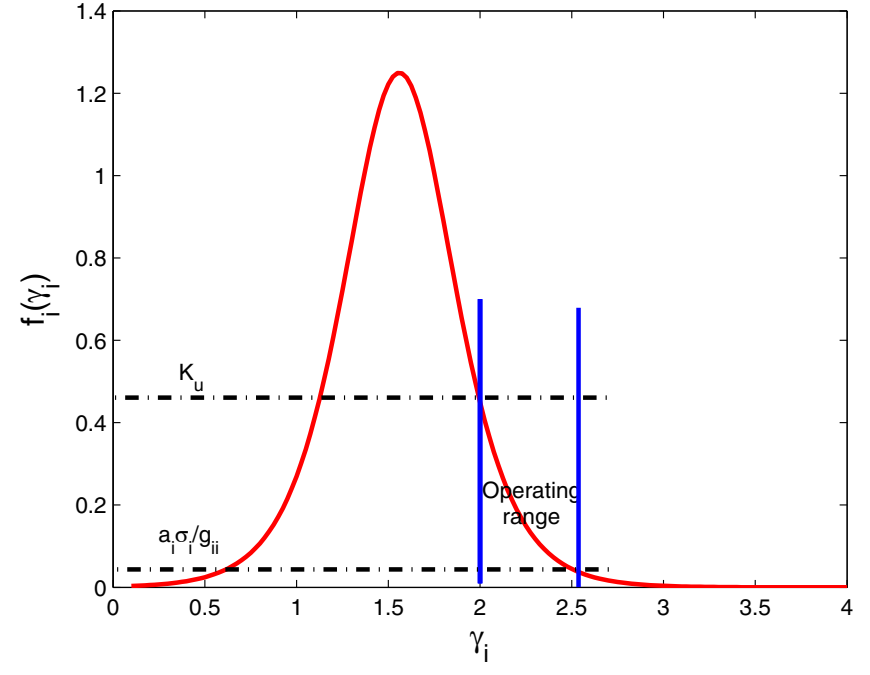

Fig. 2. Illustration of the equilibrium solution for $\Gamma_{i}=2, b_{i}=5, K_{u}=$ $U_{i}^{\prime}\left(\Gamma_{i}\right), a_{i}=a_{i}^{(m)}$.

\section{B. Dynamic Pricing, Power Adaptation and Admission Con- trol of Femtocell Users}

Given the MUEs' QoS requirements already supported, the specific choice of utility and cost functions for FUEs allows us to achieve several practical design objectives, through which certain user satisfaction metrics can be attained. Notice that if the FUEs also wish to maintain their respective QoS requirements, the operation of these users may cause network congestion, hence badly affecting the performance of the MUEs. In such cases, the FUEs should be penalized by appropriately regulating their operating parameters. Motivated by the above observation, we will now describe two design options for the FUEs, each with a different design objective. Under each option, we will propose a joint power adaptation and admission control algorithm. The convergence of the developed solutions will be analyzed, followed by the characterization of their corresponding equilibria.

1) Balancing Achieved Throughput and Power Expenditure for Femtocell Users: We choose a utility function that captures the Shannon capacity for the FUEs, $U_{i}\left(\gamma_{i}\right)=W \ln \left(1+\gamma_{i}\right)$ where $W$ denotes the system bandwidth, and a linear cost function $C\left(p_{i}\right)=a_{i}^{(f)} p_{i}$ with pricing coefficient $a_{i}^{(f)}$. Altogether, the net utility for FUE $i$ is defined as

$$
U_{\text {tot }, i}=W \ln \left(1+\gamma_{i}\right)-a_{i}^{(f)} p_{i}, \quad \forall i \in \mathcal{L}_{f} .
$$

Such choices of functions are especially relevant when the FUEs have to tradeoff between achieving the highest possible data rates and expending as little power as necessary. Applying the result in (7) to these utility and cost functions gives

$$
\frac{W}{1+\gamma_{i}}=\frac{a_{i}^{(f)} I_{i}}{g_{i i}}
$$

From (22) and upon noting that $U_{\text {tot }, i}$ is strictly concave in $p_{i}$, the value of $p_{i} \geq 0$ that globally maximizes $U_{\mathrm{tot}, i}$ can be derived as

$$
p_{i}^{*}=\max \left(\frac{W}{a_{i}^{(f)}}-\frac{I_{i}}{g_{i i}}, 0\right)
$$




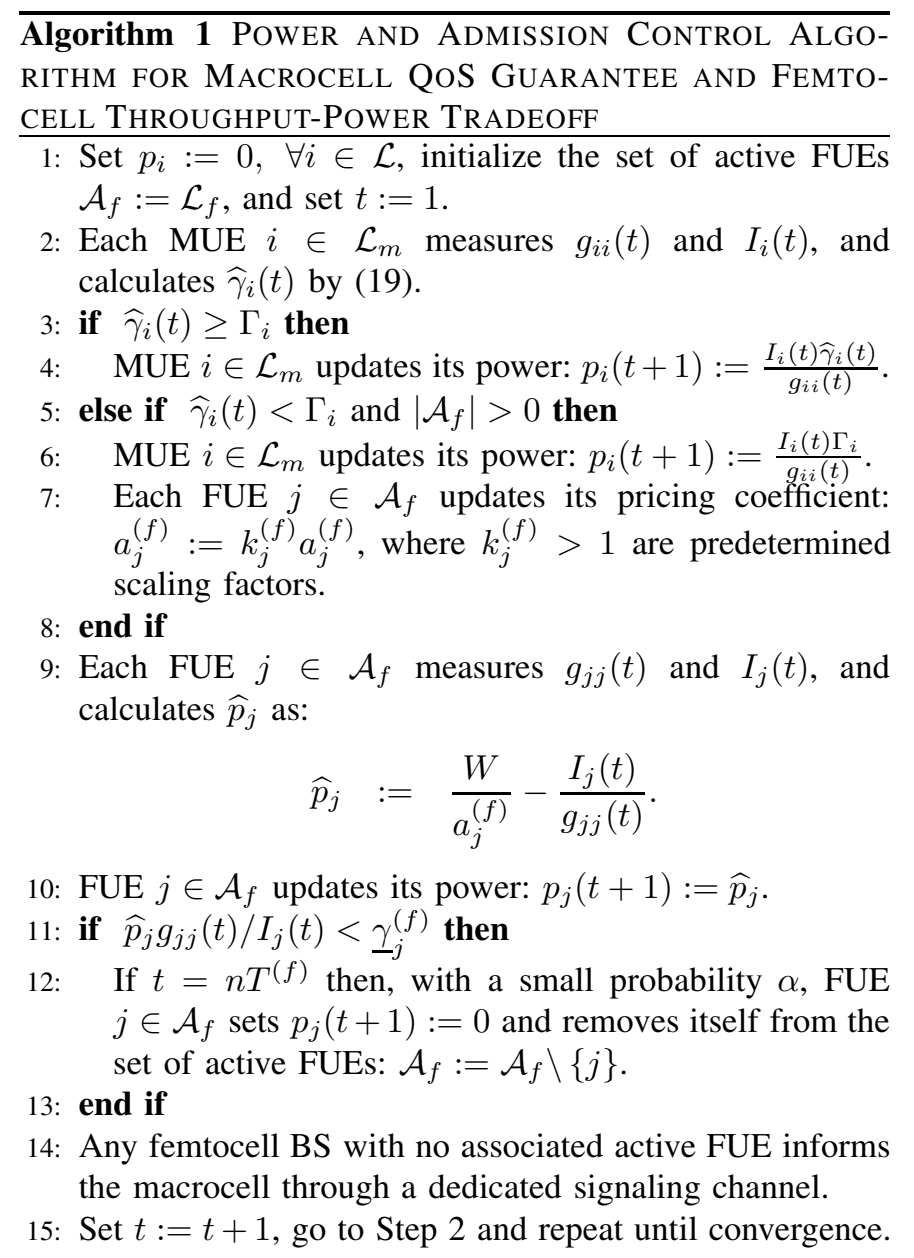

We present in Algorithm 1 a joint power and admission control scheme for the interference management in both macrocell and femtocell networks. This algorithm lends itself to a distributed implementation with only local information required. In every iteration, each user $i \in \mathcal{L}$ simply needs to estimate (i) its received interference power $I_{i}(t)$ and (ii) its own channel gain $g_{i i}(t)$ in order to update its transmit power. When there exists an active MUE $i$ with its "soft" SINR target $\widehat{\gamma}_{i}(t)$ dropping below the prescribed SINR target $\Gamma_{i}$, we gradually increase the pricing coefficients $a_{j}^{(f)}$ of all the active FUEs [see Step 7]. It is apparent from (23) that such an increase in $a_{j}^{(f)}$ results in a reduction in the transmit power of FUE $j$, through which the user that creates undue cross-tier interference can be effectively penalized. Notably, this pricing mechanism is realized without acquiring the knowledge of cross-channel gains, unlike the one proposed by [28].

The procedure of updating the pricing coefficients described in Step 7 of Algorithm 1 impacts both the convergence speed of that algorithm and the number of active FUEs at the resulting equilibrium. A higher initial pricing $a_{j}^{(f)}$ and/or a larger scaling factor $k_{j}^{(f)}>1$ will shorten the convergence time, albeit at the cost of being able to support a fewer number of active FUEs at the equilibrium point. Therefore, careful selections of $a_{j}^{(f)}$ and $k_{j}^{(f)}$ to reflect the relative amount of interference that FUE $j \in \mathcal{L}_{f}$ induces to other users may lead to better network performance. In particular, it is sensible to set large values of $a_{j}^{(f)}$ and $k_{j}^{(f)}$ for the FUE $j$ that creates excessive interference. Eventually, these "bad" users will at least see their transmit power reduced at equilibrium. In networks with a high load level ${ }^{1}$, they can even be removed, through which the built-up network congestion is relieved.

In Step 12 of Algorithm 1, if a certain FUE $j$ has its SINR falling below the minimum required threshold $\underline{\gamma}_{j}^{(f)}$, it is removed with probability $\alpha$ (where $0<\alpha<1$ ) at most once in every $T^{(f)}$ iterations. Note that a smaller value of $\alpha$ will prevent the unnecessary elimination of too many FUEs, at the expense of prolonging the convergence time. The same effect can also be expected for large values of $T^{(f)}$.

Theorem 1: The proposed Algorithm 1 converges to an equilibrium solution if $x f_{i}^{-1}(x)$ is an increasing function, $\forall i \in \mathcal{L}_{m}$, and the following condition

$$
\left(M_{m}+N_{f}-1\right) R_{\max }<1
$$

holds, where $f_{i}(\cdot)=U_{i}^{\prime}(\cdot) ; M_{m}=\left|\mathcal{A}_{m}\right| \leq M$ and $N_{f}=$ $\left|\mathcal{A}_{f}\right| \leq N$ denote the cardinality of the active macrocell and femtocell user sets, respectively; and $R_{\max }$ is defined as

$$
R_{\max }=\max _{\left\{i \in \mathcal{L}_{f}, j \in \mathcal{L} \backslash\{i\}\right\}} \frac{g_{i j}}{g_{i i}}=\max _{\left\{i \in \mathcal{L}_{f}, j \in \mathcal{L} \backslash\{i\}\right.} \frac{g_{i j}}{G g_{i i}^{\prime}} .
$$

Moreover, for users who achieve nonzero powers at the equilibrium, it is true that

$$
\begin{aligned}
p_{i}^{*} & =\frac{I_{i}^{*}}{g_{i i}} f_{i}^{-1}\left(\frac{a_{i}^{(m)} I_{i}^{*}}{g_{i i}}\right), i \in \mathcal{A}_{m}, \\
p_{i}^{*} & =\frac{W}{a_{i}^{(f)}}-\frac{I_{i}^{*}}{g_{i i}}, i \in \mathcal{A}_{f},
\end{aligned}
$$

where $I_{i}^{*}=\sum_{j \neq i} g_{i j} p_{j}^{*}+\sigma_{i}$. Further, all active MUEs $i \in \mathcal{A}_{m}$ have their SINR $\gamma_{i}^{*}$ satisfying $\gamma_{i}^{*} \geq \Gamma_{i}$.

Proof: The proof can be found in Appendix A.

2) Soft QoS Provisioning for Femtocell Users: In this scenario, we assume that FUE $i \in \mathcal{L}_{f}$ also requires a minimum SINR $\Gamma_{i}$ to maintain the quality of its applications. Note that the meaning of $\Gamma_{i}$ here is very different from that of $\underline{\gamma}_{i}^{(f)}$ defined in (4). In practice, the value of $\Gamma_{i}$ is typically greater than $\underline{\gamma}_{i}^{(f)}$. While a higher SINR at the receiving end of any femto links implies more reliability and better services, this usually requires more transmit power, which in turn leads to a higher cross-interference induced to the macrocell. Such an observation motivates us to consider the following net utility for FUE $i$ (similar to that in [31]):

$$
U_{\text {tot }, i}=-\left(\gamma_{i}-\Gamma_{i}\right)^{2}-a_{i}^{(f)} p_{i}, \quad \forall i \in \mathcal{L}_{f}
$$

Although maximizing the first term on the right-hand side of the above equation, i.e., the utility $U_{i}\left(\gamma_{i}\right)=-\left(\gamma_{i}-\Gamma_{i}\right)^{2}$, enforces the SINR $\gamma_{i}$ of FUE $i$ to be as close as possible to

\footnotetext{
${ }^{1}$ In this paper, the network load is defined to be "low" if $\bar{\rho}=$ $\rho\left(\operatorname{diag}\left(\left[\boldsymbol{\Gamma}_{m} ; \boldsymbol{\Gamma}_{f}\right]\right) \overline{\mathbf{G}}\right)<1$, where $\rho(\cdot)$ denotes the matrix spectral radius, $\overline{\mathbf{G}}=\left[g_{i j}\right]$ is the channel gain matrix, and $\boldsymbol{\Gamma}_{m}$ and $\boldsymbol{\Gamma}_{f}$ are the vectors of minimum SINRs required by the MUEs and the FUEs, respectively. When $\bar{\rho} \geq 1$, not all these minimum SINRs can be supported with a finite amount of transmit power [30]. In such a case, the network load level is either "medium" or "high" depending on the specific value of $\bar{\rho}$, and admission control is needed to remove some FUEs. If $\bar{\rho}$ tends to be much larger than 1 , the network becomes very congested where it is even difficult to support the minimum SINR $\boldsymbol{\Gamma}_{m}$ of the prioritized MUEs alone.
} 
the SINR target $\Gamma_{i}$, the resulting $\gamma_{i}^{*}$ at the equilibrium may actually be less than $\Gamma_{i}$. Nevertheless, it has been shown in [31] that by allowing a reasonable deviation from the target SINR, a significant reduction in the transmit power (and hence the resulting interference) can be achieved. Given its lower access priority, this type of soft QoS provisioning is totally acceptable for FUE $i$. On the other hand, the cost function, $C_{i}\left(p_{i}\right)=a_{i}^{(f)} p_{i}$, penalizes the expenditure of transmit power, which potentially creates undue interference to the macrocell as well as other FUEs. Here, $a_{i}^{(f)}$ is the pricing coefficient of such penalization.

Now, applying the result in (7) to these particular utility and cost functions yields:

$$
\gamma_{i}=\Gamma_{i}-\frac{a_{i}^{(f)} I_{i}}{2 g_{i i}}
$$

Because $U_{i}\left(\gamma_{i}\right)$ is a concave function in $p_{i}$, so is $U_{\text {tot }, i}, \forall i \in$ $\mathcal{L}_{f}$. The power value that globally maximizes $U_{\text {tot }, i}$ can thus be computed as

$$
p_{i}^{*}=\max \left(\frac{I_{i} \Gamma_{i}}{g_{i i}}-\frac{a_{i}^{(f)} I_{i}^{2}}{2 g_{i i}^{2}}, 0\right) .
$$

Again, by setting the pricing coefficient $a_{i}^{(f)}$ sufficiently large, we can effectively shut off FUE $i$. Based upon the power update rule in (30), a joint power adaptation and admission control algorithm is now developed that is capable of providing soft QoS for the FUEs. This algorithm is referred to as Algorithm 2 in the sequel. The steps in Algorithm 2 are identical to those in Algorithm 1 except for Step 9 where $\widehat{p}_{j}$ is, instead, calculated as $\widehat{p}_{j}=I_{j}(t) \Gamma_{j} / g_{j j}(t)-$ $a_{j}^{(f)} I_{j}^{2}(t) /\left[2 g_{j j}^{2}(t)\right], \forall j \in \mathcal{A}_{f}$.

Theorem 2: Assuming that $x f_{i}^{-1}(x)$ is an increasing function, $\forall i \in \mathcal{L}_{m}$, the proposed Algorithm 2 converges to an equilibrium, at which point

$$
\begin{aligned}
& p_{i}^{*}=\frac{I_{i}^{*}}{g_{i i}} f_{i}^{-1}\left(\frac{a_{i}^{(m)} I_{i}^{*}}{g_{i i}}\right), i \in \mathcal{A}_{m} \\
& p_{i}^{*}=\frac{I_{i}^{*} \Gamma_{i}}{g_{i i}}-\frac{a_{i}^{(f)}\left(I_{i}^{*}\right)^{2}}{2 g_{i i}^{2}}, i \in \mathcal{A}_{f} .
\end{aligned}
$$

Moreover, all active MUEs $i \in \mathcal{L}_{m}$ have their SINR $\gamma_{i}^{*}$ satisfying $\gamma_{i}^{*} \geq \Gamma_{i}$.

Proof: The proof can be found in Appendix B.

\section{Practical Implementation Issues and Further EXTENSIONS}

\section{A. Communication Overhead of the Proposed Algorithms}

The schemes developed in this work only require a limited amount of signaling to be exchanged among the femtocells and the macrocell. In either algorithm, the power updates of both MUEs and FUEs can be executed in a completely distributed manner, based on the information available at local links. On one hand, the receiver of each user $i$ (i.e., either the BS or the user terminal depending on uplink or downlink transmission, respectively) can estimate $g_{i i}^{\prime}$ by, for instance, exploiting the pilot channel. On the other hand, this receiver can also measure the total received power, and then subtract its own received power to obtain the aggregated interference
$I_{i}$, i.e., $I_{i}=\sum_{j \in \mathcal{L}} g_{i j} p_{j}-g_{i i}^{\prime} p_{i}$, assuming that noise can be ignored in interference-limited CDMA links. The receiver of user $i$ then sends both values of $g_{i i}^{\prime}$ and $I_{i}$ to its corresponding transmitter for the update of transmit power in each iteration.

In Step 7 of Algorithms 1 and 2, the FUEs are requested to increase their pricing coefficients when certain MUEs perceive network congestion. Apparently, each MUE may only experience significant interference from the FUEs within its immediate neighborhood. To protect the MUEs in the downlink case, it would therefore be sufficient that only the macrocell receivers with low SINRs request their neighboring FUEs to increase their pricing coefficients. In case of open access, the hand-off procedure should be established between the users and the macrocell/femtocell BSs, with a control channel dedicated for this purpose. Hence, the "warning" message that asks for an increase in the FUEs' prices can be incorporated into the hand-off message when the undue crosstier interference is sensed by the victim MUEs. The other type of communication overhead includes the notification made by the femtocell BS that serves no FUEs to the macrocell in Step 14 of Algorithms 1 and 2 . This may take the form of a simple flag message, to be sent over the available wireline backhaul network or be broadcast wirelessly.

\section{B. Improving the Efficiency of Equilibrium Solution}

It can be shown that the equilibrium solutions achieved by the developed algorithms correspond to the Nash equilibria of the underlying non-cooperative games [32]. In such states, no user has any incentive to unilaterally change its transmit power level. However, Nash equilibrium in general does not guarantee to be either globally efficient or optimal. We discuss here a mechanism to improve the efficiency of this equilibrium, particularly when the network is lightly loaded. Specifically, we attempt to make the SINRs of the active MUEs greater than their required SINRs. In wireless environments, this result implies more service reliability and more robustness against fading for the MUEs.

From (13), the following relationship at the equilibrium can be obtained for the active MUE $i$ :

$$
f_{i}\left(\gamma_{i}^{*}\right)=\frac{a_{i}^{(m)} I_{i}^{*}}{g_{i i}}
$$

where the typical shape of function $f_{i}(\cdot)$ has already been illustrated in Fig. 2. It is observed that a higher SINR $\gamma_{i}^{*}$ can be realized for a given $a_{i}^{(m)} I_{i}^{*} / g_{i i}$ if $f_{i}(\cdot)$ becomes flatter. This corresponds to choosing smaller values of $b_{i}$, where recall that $b_{i}$ is the parameter that controls the steepness of $U_{i}\left(\gamma_{i}\right)$. Ultimately, it is possible that the SINRs of MUEs are enhanced by reducing $b_{i}$ whenever possible. Nevertheless, the values of $b_{i}$ should be updated less frequently compared with the update of power itself.

Toward that end, the following procedure can be employed to improve the attained SINRs of the active MUEs: We choose in advance a particular interval $T_{b}$ to periodically update $b_{i}, \forall i \in \mathcal{L}_{m}$. At the beginning of each interval $T_{b}$, MUE $i \in \mathcal{L}_{m}$ multiplies its $b_{i}$ by a factor $k_{b}<1$ if its servicing macrocell BS has not been informed about any empty femtocell during the previous interval. The latter 
TABLE I

SIMULATION PARAMETERS

\begin{tabular}{r||l}
\hline Parameter & Value \\
\hline \hline Path-loss exponent, $\beta$ & 3 \\
\hline Processing gain, $G$ & 100 \\
\hline Noise power, $\sigma_{i}=\sigma$ (in Watt) $\forall i \in \mathcal{L}$ & $10^{-10}$ \\
\hline System bandwidth, $W$ (in Hertz) & $10^{6}$ \\
\hline$\underline{\gamma}_{i}^{(f)}=\gamma^{(f)} \quad \forall i \in \mathcal{L}_{f}$ & 2 \\
\hline$a_{i}^{(m)}=a_{i} \quad \forall i \in \mathcal{L}_{m}$ & 1 \\
\hline$b_{i}$ & 1 \\
\hline Removal probability, $\alpha$ & 0.1 \\
\hline$T^{(f)}$ & 10 \\
\hline$T_{b}$ & 20 \\
\hline$k_{b}$ & 0.5 \\
\hline
\end{tabular}

condition happens if the network load is low, which also means that almost all the FUEs converge to the desired equilibrium without being removed.

\section{Maximum Power Constraints}

In the previous sections, we have assumed that both the MUEs and the FUEs can transmit at arbitrarily large power levels. We now discuss the scenarios wherein the users are subject to power limits of the form $0 \leq p_{i} \leq p_{i}^{\max }, \forall i \in \mathcal{L}$. Here, the power updates of both MUEs and FUEs are performed according to $p_{i}(t+1):=\min \left\{p_{i}^{\max }, \widehat{p}_{i}(t+1)\right\}$, with $\widehat{p}_{i}(t+1)$ the power assignment in the case of no maximum power constraint imposed. Fortunately, the convergence results of both Theorems 1 and 2 still hold true because (i) standard functions with upper power constraints remain standard [33], and (ii) inequality (38) is valid even with constrained powers. Nonetheless, as the transmit power budget is limited in this case, the achieved SINRs of the MUEs at the equilibrium may drop below their minimum requirements. If certain MUEs achieve very low SINRs at the equilibrium while there is no active FUE remaining in the system, we may allow these MUEs to remove themselves with a small probability. As soon as the network congestion is sufficiently relieved via this removal process, all the remaining active MUEs will eventually meet their SINR targets.

\section{NumericAl Results}

This section presents numerical results to demonstrate the performance of the proposed Algorithms 1 and 2. The network setting and user placement in these examples are illustrated in Fig. 1, where the MUEs and the FUEs are randomly deployed inside circles of radii of $500 \mathrm{~m}$ and 100 $\mathrm{m}$, respectively. Downlink transmission is considered in all the simulations. We also assume that the number of FUEs serviced by any femtocell BS is identical. The specific numbers of MUEs and FUEs generated in each example are displayed in the corresponding plot. The results presented in each figure correspond to one particular network realization, chosen with the intention to demonstrate certain features of the developed algorithms.

The channel gain from the transmitter of user $j$ to the receiver of user $i$ is calculated as $d_{i j}^{-\beta}$, where $d_{i j}$ is their

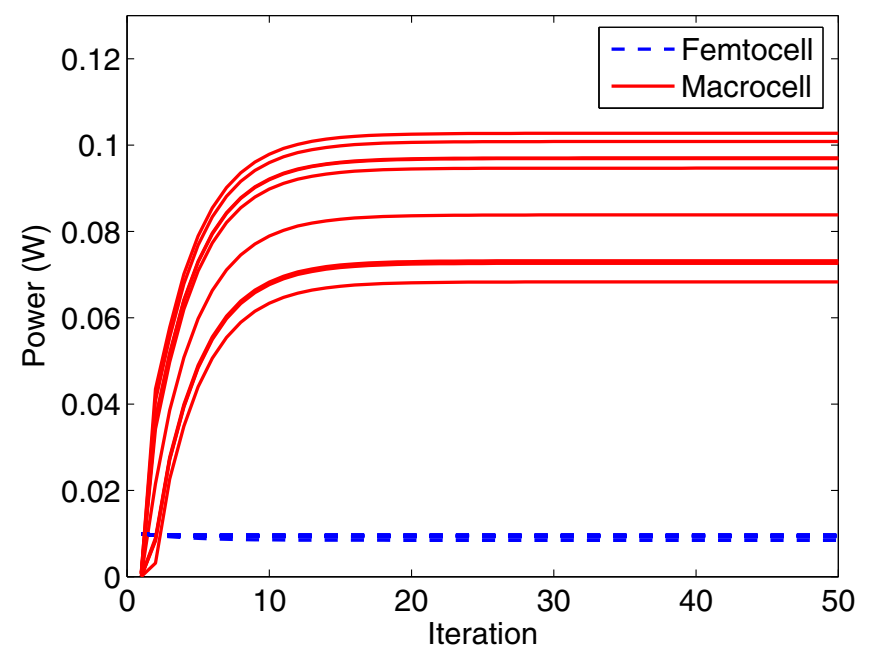

Fig. 3. Algorithm 1: Power evolution for $M=10, N=20, \Gamma_{i}^{(m)}=$ $8, a^{(f)}=10^{9}$, and $k^{(f)}=1.1$.

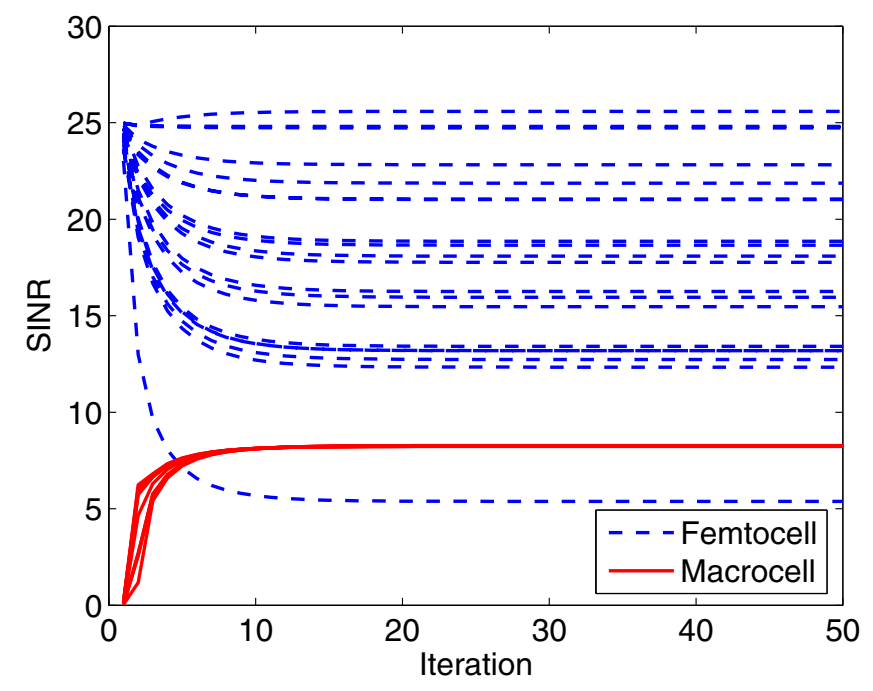

Fig. 4. Algorithm 1: SINR evolution for $M=10, N=20, \Gamma_{i}^{(m)}=$ $8, a^{(f)}=10^{9}$, and $k^{(f)}=1.1$.

geographical distance and $\beta$ the pathloss exponent. The same initial pricing coefficient $a_{i}^{(f)}=a^{(f)}$ and scaling parameter $k_{i}^{(f)}=k^{(f)}$ are used for all the FUEs. Their values, together with the SINR targets $\Gamma_{i}^{(m)}$ and $\Gamma_{i}^{(f)}$, can be found underneath every plot. In each figure, a single curve corresponds to one specific user. For the ease of reference, the simulation parameters are summarized in Table I.

In Figs. 3 and 4, we show the evolutions of powers and SINRs under Algorithm 1. As can be seen, Algorithm 1 converges to an equilibrium with the target SINRs being attained for all the MUEs. It is also clear from these figures that the convergence time of Algorithm 1 is relatively short, slightly larger than 10 in this case. On the other hand, Fig. 5 illustrates the operation of Algorithm 1 when the network becomes congested. This algorithm initially converges to an equilibrium in which the SINR requirement of one FUE cannot be satisfied, i.e., its final SINR drops below the threshold $\gamma^{(f)}=2$. Then, the admission control mechanism integrated in Algorithm 1 is engaged to effectively remove this user, 


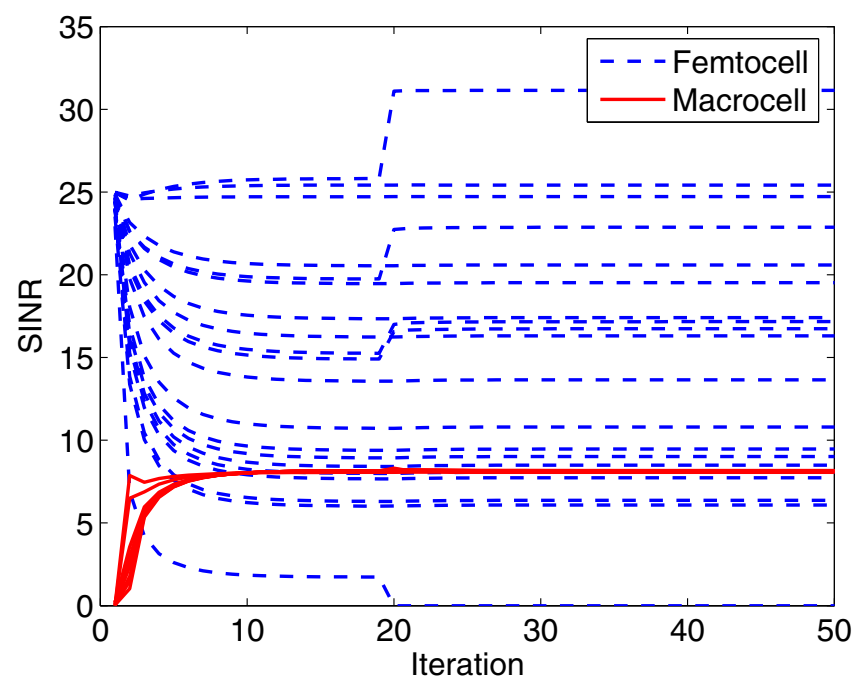

Fig. 5. Algorithm 1: SINR evolution with FUE removal for $M=10, N=$ $20, \Gamma_{i}^{(m)}=8, a^{(f)}=10^{9}$, and $k^{(f)}=1.1$.

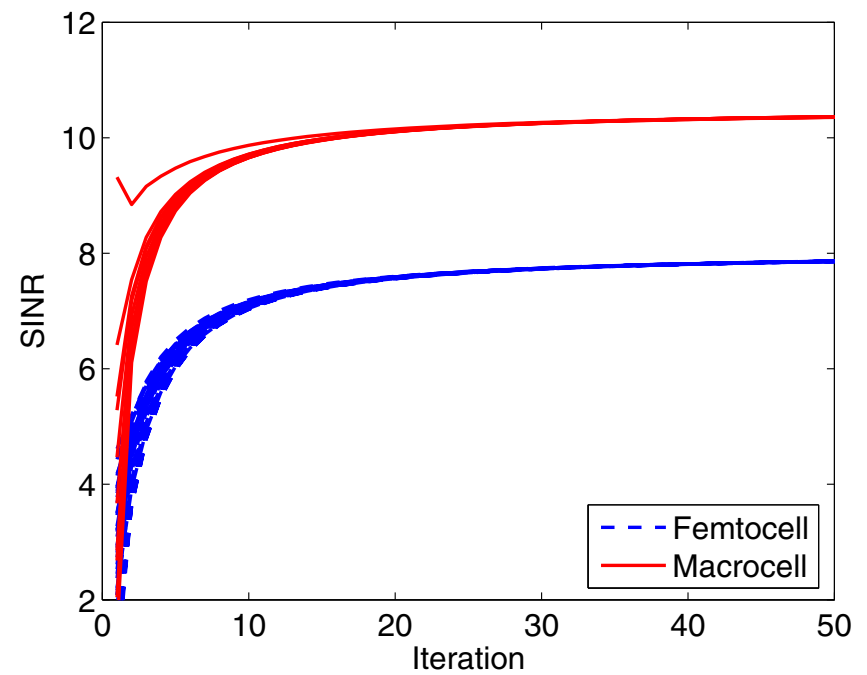

Fig. 6. Algorithm 2: SINR evolution in low load (i.e., $\left.\rho\left(\operatorname{diag}\left(\left[\boldsymbol{\Gamma}_{m} ; \boldsymbol{\Gamma}_{f}\right]\right) \overline{\mathbf{G}}\right)=0.9\right)$ for $M=10, N=40, \Gamma_{i}^{(m)}=$ $10, \Gamma_{i}^{(f)}=8, a^{(f)}=10^{4}$, and $k^{(f)}=1.5$.

resulting in a noticeable growth in SINRs of several other FUEs [see iteration 20 and beyond]. It is also evident here that the removal of the FUEs does not affect the transmit powers and SINRs of the MUEs. This result verifies the efficiency and robustness of Algorithm 1 in protecting the macrocell performance.

In Figs. 6, 7, and 8, we display the evolutions of SINRs for all the users under Algorithm 2 when the network load level is low, medium, and high, respectively. In all the scenarios, it is confirmed that Algorithm 2 actually converges with the SINR requirements of all MUEs being met at the equilibrium. When the network becomes more congested, the convergence speed appears to be slower. Specifically, Fig. 6 shows that when the network load is low, the achieved SINRs of the FUEs are slightly below their corresponding requirements while the performance of all MUEs is well protected. This is a desirable feature as the soft QoS for the lower-tier FUEs

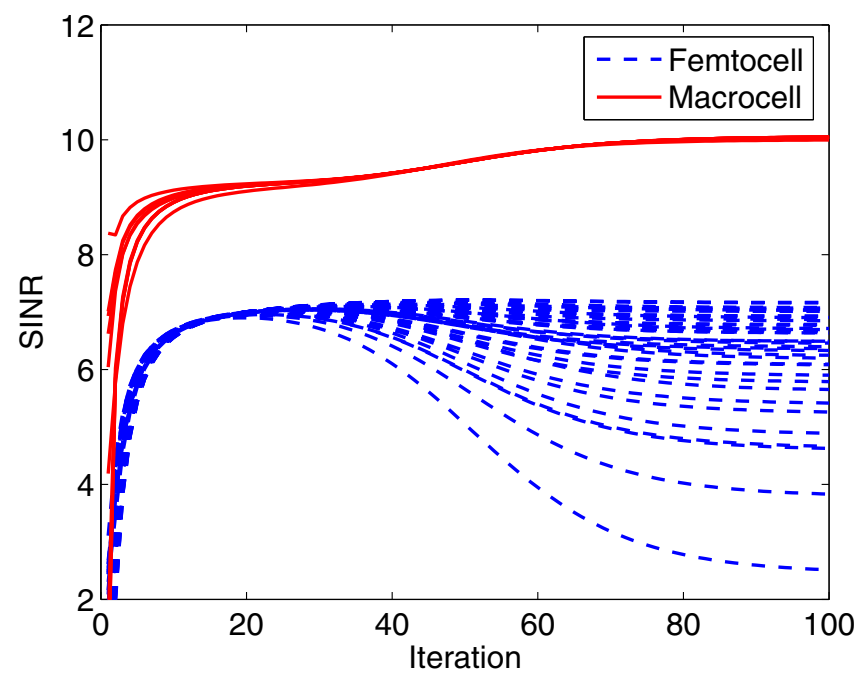

Fig. 7. Algorithm 2: SINR evolution in medium load (i.e., $\left.\rho\left(\operatorname{diag}\left(\left[\boldsymbol{\Gamma}_{m} ; \boldsymbol{\Gamma}_{f}\right]\right) \overline{\mathbf{G}}\right)=1.1\right)$ for $M=10, N=40, \Gamma_{i}^{(m)}=10, \Gamma_{i}^{(f)}=$ $8, a^{(f)}=10^{4}$, and $k^{(f)}=1.5$.

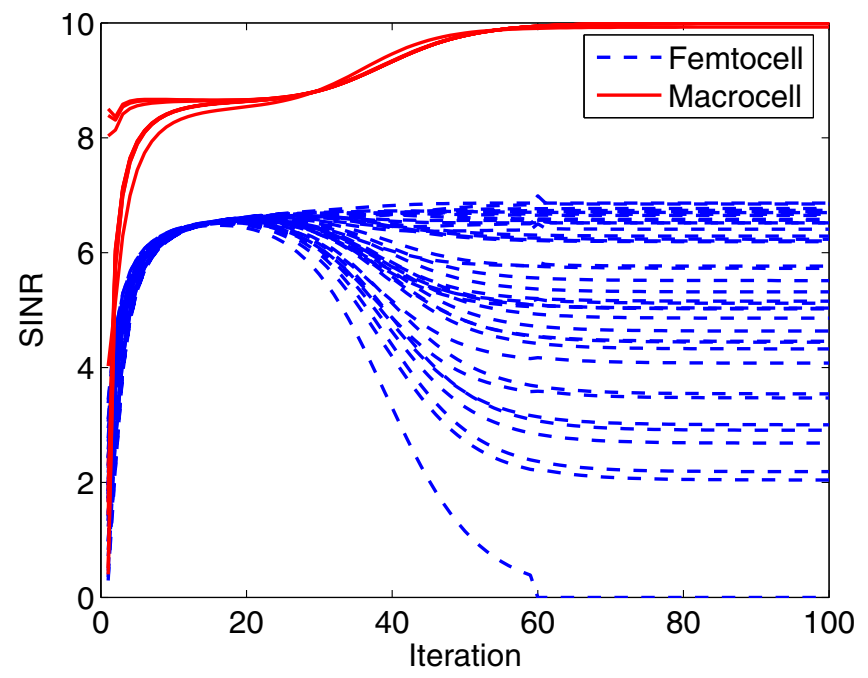

Fig. 8. Algorithm 2: SINR evolution in high load (i.e., $\left.\rho\left(\operatorname{diag}\left(\left[\boldsymbol{\Gamma}_{m} ; \boldsymbol{\Gamma}_{f}\right]\right) \overline{\mathbf{G}}\right)=1.18\right)$ for $M=10, N=40, \Gamma_{i}^{(m)}=$ $10, \Gamma_{i}^{(f)}=8, a^{(f)}=10^{4}$, and $k^{(f)}=1.5$.

can only be supported to the extent that network load allows. When network congestion starts building up, Algorithm 2 smoothly reduces the SINRs of the FUEs so that the MUEs can eventually reach their desired SINR targets. This feature can best be observed in Fig. 7. Finally, when the network gets so congested that the SINRs of certain FUEs fall below the minimum required threshold $\underline{\gamma}^{(f)}=2$, admission control is executed to remove such users. This operation is depicted in Fig. 8, where the FUE that achieves the smallest SINR value is eliminated from the network.

Fig. 9 illustrates how the technique presented in Section IV-B can help improve the achieved SINRs of the MUEs in Algorithm 1. Recall that such a mechanism, which involves scaling down the values of $b_{i}$ over time, may only be activated when the network load level is low. To obtain the results presented in this figure, we have decreased all $b_{i}$ 's by a factor $k_{b}=0.5$ once in every $T_{b}=20$ iterations. These updates 


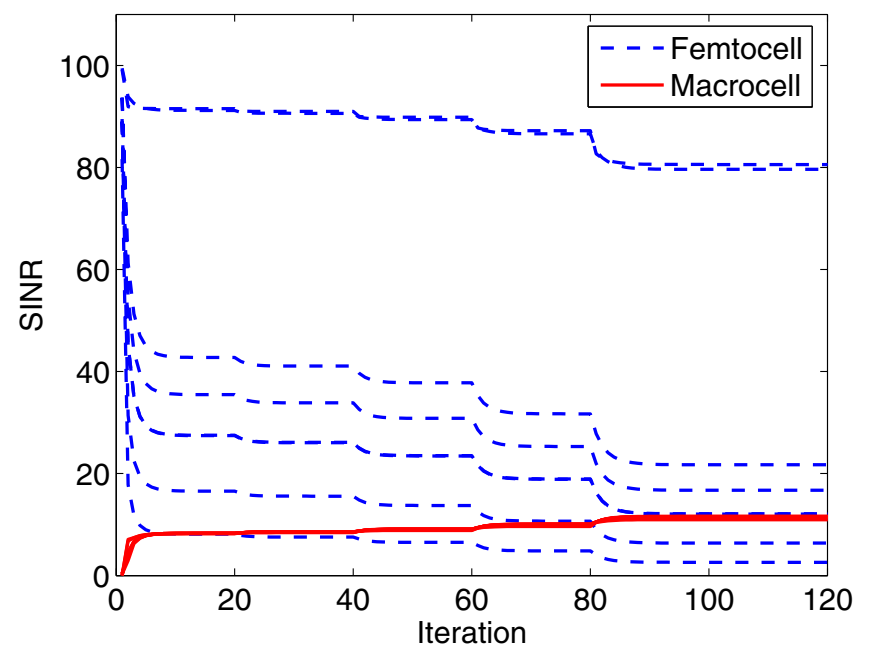

Fig. 9. Algorithm 1: Improving SINRs of the MUEs for $M=8, N=$ $8, \Gamma_{i}^{(m)}=8, a^{(f)}=10^{9}, k^{(f)}=1.1$ and $k_{b}=0.5$.

are carried on until one FUE settles its SINR below the specified SINR threshold $\gamma^{(f)}=2$. Furthermore, $T_{b}$ is set to be sufficiently large so that the algorithm converges to a new equilibrium. As shown in Fig. 9, by scaling down $b_{i}$, we can enhance the attained SINRs of all the MUEs at the cost of degrading the SINRs of the FUEs.

\section{CONCLUSION}

In this paper, we have proposed joint power adaptation and admission control algorithms to autonomously manage the interference in two-tier networks. Specifically, two different design options for the FUEs have been considered: (i) Balancing between their achieved throughput and the expended power, and (ii) Supporting their soft QoS. It has been shown that the developed algorithms are able to robustly protect the MUEs by maintaining their desired SINR requirements, while also allowing the FUEs to flexibly share the remaining network capacity. Upon applying the proposed dynamic pricing scheme combined with admission control, network congestion can be effectively alleviated whenever necessary. The convergence of the developed solutions has been proved analytically, and their merits are confirmed through extensive numerical study.

\section{APPENDIX A}

\section{PROOF OF THEOREM 1}

First, the power control updates for the MUEs and the FUEs can be summarized as:

$$
\mathbf{p}(t+1)=\left[A_{i}(\mathbf{p}(t))\right], i \in \mathcal{L},
$$

where, specifically,

$$
A_{i}(\mathbf{p}(t))=\max \left\{\frac{I_{i}(t) \Gamma_{i}}{g_{i i}(t)}, \frac{I_{i}(t)}{g_{i i}(t)} f_{i}^{-1}\left(\frac{a_{i}^{(m)} I_{i}(t)}{g_{i i}(t)}\right)\right\},
$$

for $i \in \mathcal{L}_{m}$, and

$$
A_{i}(\mathbf{p}(t))=\max \left\{\frac{W}{a_{i}^{(f)}}-\frac{I_{i}(t)}{g_{i i}(t)}, 0\right\},
$$

for $i \in \mathcal{L}_{f}$.
Let us define $\Delta p_{i}(t)=p_{i}(t)-p_{i}^{*}$ with $p_{i}^{*}$ being the transmit power of user $i$ at the equilibrium [see (26) and (27)]. Also, denote by $\|\Delta \mathbf{p}\|_{\mathcal{A}}$ the $l_{\infty}$-norm of vector $\Delta \mathbf{p}$ over some set $\mathcal{A}$, i.e., $\|\Delta \mathbf{p}\|_{\mathcal{A}}=\max _{i \in \mathcal{A}}\left|\Delta p_{i}\right|$. Upon applying the results in (36) and (27) to a particular FUE $i \in \mathcal{A}_{f}$, we have the following:

$$
\begin{aligned}
\left|\Delta p_{i}(t+1)\right| & =\left|\frac{I_{i}^{*}-I_{i}(t)}{g_{i i}}\right| \\
& \leq(1 / G)\left|\sum_{j \neq i}\left(g_{i j} / g_{i i}^{\prime}\right) \Delta p_{j}(t)\right| \\
& \leq(1 / G) \sum_{j \neq i}\left|\left(g_{i j} / g_{i i}^{\prime}\right) \Delta p_{j}(t)\right| \\
& \leq R_{\max } \sum_{j \neq i}\left|\Delta p_{j}(t)\right| \\
& \leq R_{\max }\left(M_{m}+N_{f}-1\right)\|\Delta \mathbf{p}(t)\|_{\mathcal{A}_{f} \cup \mathcal{A}_{m}}
\end{aligned}
$$

From this, it is clear that

$$
\|\Delta \mathbf{p}(t)\|_{\mathcal{A}_{f}} \leq\left(M_{m}+N_{f}-1\right) R_{\max }\|\Delta \mathbf{p}(t)\|_{\mathcal{A}_{f} \cup \mathcal{A}_{m}} .
$$

Hence, $\|\Delta \mathbf{p}(t)\|_{\mathcal{A}_{f}}$ will shrink over time if the condition in (24) is satisfied and also if $\|\Delta \mathbf{p}(t)\|_{\mathcal{A}_{m}}$ shrinks over time.

The inequality $\left(M_{m}+N_{f}-1\right) R_{\max }<1$ in (24) is usually met if the network is not very congested. Here, because the direct channel gains dominate the cross-channel gains, $R_{\max }$ typically takes small values. In the case of network congestion, the admission control in Step 12 of Algorithm 1 will remove several FUEs (i.e., decreasing $N_{f}$ ), making it possible to fulfill such a condition. Even for very dense femtocell/macrocell deployment, (24) can still be satisfied by using a large value of processing gain $G$, through which $R_{\max }$ can be made small. As well, the integrated admission control mechanism in Algorithm 1 will autonomously remove "bad users" if the network becomes congested, making (24) feasible.

On the other hand, it has been shown in [20] that $A_{i}(\mathbf{p}(t)), i \in \mathcal{L}_{m}$ in (35) is a standard function (i.e., it satisfies the positivity, monotonicity, and scalability attributes [33]) if $x f_{i}^{-1}(x)$ is an increasing function. Moreover, [33] has established that a power control algorithm will converge if such standard function properties are satisfied. It can be verified that $x f_{i}^{-1}(x)$ is an increasing function if $b_{i}$ is sufficiently large. As discussed earlier, this can certainly be achieved by our design. Since $\|\Delta \mathbf{p}(t)\|_{\mathcal{A}_{m}}$ shrinks over time, so does $\|\Delta \mathbf{p}(t)\|_{\mathcal{A}_{f}}$. As a consequence, Algorithm 1 converges to an equilibrium.

Furthermore, all active MUEs $i \in \mathcal{A}_{m}$ at that equilibrium state must have their SINR $\gamma_{i}^{*}$ satisfying $\gamma_{i}^{*} \geq \Gamma_{i}$. This is the case because all of the remaining users in the equilibrium must be admissible; otherwise, some of them must have been removed by the admission control mechanism integrated in Algorithm 1.

\section{APPENDIX B}

\section{PROOF OF THEOREM 2}

The convergence of the proposed power updates in this case can be proven using the standard function technique [33]. For the MUEs, [20] maintains that $A_{i}(\mathbf{p}(t)), i \in \mathcal{L}_{m}$ is a standard 
function if $x f_{i}^{-1}(x)$ is an increasing function. As discussed in the proof of Theorem 1 , this is true if $b_{i}$ is chosen to be sufficiently large.

For the FUEs, it has been shown in [31] that the power updates for such users [see (30)] satisfy the requirements of a standard function if the following conditions hold for all $i \in \mathcal{L}_{f}$ :

$$
\begin{aligned}
I_{i} & <\frac{g_{i i} \Gamma_{i}}{a_{i}^{(f)}}, \\
p_{i} & \leq \frac{\Gamma_{i}^{2}}{2 a_{i}^{(f)}} .
\end{aligned}
$$

Indeed, conditions (39)-(40) can be enforced by the admission control mechanism in Algorithm 2. If the network is congested enough, the transmit powers of certain users will diverge to some large values, creating a large amount of interference $I_{i}(t)$ to other users. Note that the power update for $i \in \mathcal{L}_{f}$ satisfies $\gamma_{i}(t+1)=\Gamma_{i}-a_{i}^{(f)} I_{i}(t) /\left(2 g_{i i}\right)$. Therefore, if $I_{i}(t)$ is sufficiently large so that $\gamma_{i}(t+1)<\underline{\gamma}_{i}^{(f)}$, FUE $i$ will be removed, which in turn relieves the network congestion. Together with the proper tuning of pricing coefficient $a_{i}^{(f)}$, (39) and (40) are eventually satisfied.

Since the power updates of both the MUEs and the FUEs are standard functions, Algorithm 2 converges to an equilibrium. By the similar arguments used in the proof of Theorem 1, all active MUEs $i \in \mathcal{A}_{m}$ at that equilibrium must also have their $\operatorname{SINR} \gamma_{i}^{*} \geq \Gamma_{i}$.

\section{ACKNOWLEDGEMENT}

The authors would like to thank the anonymous reviewers, whose comments have helped improve the presentation of this paper.

\section{REFERENCES}

[1] H. Claussen, L. T. W. Ho, and L. G. Samuel, "An overview of the femtocell concept," Bell Labs. Tech. J., vol. 3, no. 1, pp. 221-245, May 2008.

[2] D. Lopez-Perez, A. Valcarce, G. de la Roche, and J. Zhang, "OFDMA femtocells: a roadmap on interference avoidance," IEEE Commun. Mag., vol. 47, no. 9, pp. 41-48, Sep. 2009.

[3] G. d. 1. Roche, A. Valcarce, D. Lopez-Perez, and J. Zhang, "Access control mechanisms for femtocells," IEEE Commun. Mag., vol. 48, no. 1, pp. 33-39, Jan. 2010.

[4] S. Kishore, L. J. Greenstein, H. V. Poor, and S. C. Schwartz, "Uplink user capacity in a CDMA system with hotspot microcells: effects of finite transmit power and dispersion," IEEE Trans. Wireless Commun., vol. 5, no. 2, pp. 417-426, Feb. 2006.

[5] V. Chandrasekhar, J. G. Andrews, and A. Gatherer, "Femtocell networks: a survey," IEEE Commun. Mag., vol. 46, no. 9, pp. 59-67, Sep. 2008.

[6] H. Claussen, "Performance of macro-and co-channel femtocells in a hierarchical cell structure," in Proc. 2007 IEEE Int. Symp. Personal, Indoor Mobile Radio Commun., pp. 1-5.

[7] I. Guvenc, M. R. Jeong, F. Watanabe, and H. Inamura, "A hybrid frequency assignment for femtocells and coverage area analysis for cochannel operation," IEEE Commun. Lett., vol. 12, no. 12, pp. 880882, Dec. 2008.

[8] D. Choi, P. Monajemi, S. Kang, and J. Villasenor, "Dealing with loud neighbors: the benefits and tradeoffs of adaptive femtocell access," in Proc. 2008 IEEE Global Telecomm. Conf.

[9] M. Yavuz, F. Meshkati, S. Nanda, A. Pokhariyal, N. Johnson, B. Raghothaman, and A. Richardson, "Interference management and performance analysis of UMTS/HSPA+ femtocells," IEEE Commun. Mag., vol. 47, no. 9, pp. 102-109, Sep. 2009.

[10] H.-S. Jo, C. Mun, J. Moon, and J.-G. Yook, "Interference mitigation using uplink power control for two-tier femtocell networks," IEEE Trans. Wireless Commun., vol. 8, no. 10, pp. 4906-4910, Oct. 2009.
[11] G. J. Foschini and Z. Miljanic, "A simple distributed autonomous power control algorithm and its convergence," IEEE Trans. Veh. Technol., vol. 42, no. 4, pp. 641-646, Nov. 1993.

[12] M. Andersin, Z. Rosberg, and J. Zander, "Gradual removals in cellular PCS with constrained power control and noise," Wireless Netw., vol. 2, pp. 27-43, 1996.

[13] N. Bambos, S. C. Chen, and G. J. Pottie, "Channel access algorithms with active link protection for wireless communication networks with power control," IEEE/ACM Trans. Netw., vol. 8, no. 5, pp. 583-597, Oct. 2000.

[14] H. Ji and C.-Y. Huang, "Non-cooperative uplink power control in cellular radio systems," Wireless Netw., vol. 4, no. 3, pp. 233-240, 1998.

[15] Z. Han and K. J. R. Liu, "Noncooperative power-control game and throughput game over wireless networks," IEEE Trans. Commun., vol. 53, no. 10, pp. 1625-1629, Oct. 2005.

[16] J. W. Lee, R. R. Mazumdar, and N. B. Shroff, "Downlink power allocation for multi-class wireless systems," IEEE/ACM Trans. Netw., vol. 13, no. 4, pp. 854-867, Aug. 2005.

[17] E. Altman, T. Boulogne, R. El-Azouzi, T. Jiminez, and L. Wynter, "A survey of network games in telecommunications," Comp. and Oper. Research, pp. 286-311, Feb. 2006.

[18] C. U. Saraydar, N. B. Mandayam, and D. J. Goodman, "Pricing and power control in a multicell wireless data network," IEEE J. Sel. Areas Commun., vol. 19, no. 10, pp. 1883-1892, Oct. 2001.

[19] _ - "Efficient power control via pricing in wireless data networks," IEEE Trans. Commun., vol. 50, no. 2, pp. 291-303, Feb. 2002.

[20] M. Xiao, N. B. Shroff, and E. K. P. Chong, "A utility-based power control scheme in wireless cellular systems," IEEE/ACM Trans. Netw., vol. 11, no. 2, pp. 210-221, Apr. 2003.

[21] M. Rasti, A. R. Sharafat, and B. Seyfe, "Pareto-efficient and goal-driven power control in wireless networks: a game-theoretic approach with a novel pricing scheme," IEEE/ACM Trans. Netw., vol. 17, no. 2, pp. 556569, Apr. 2009.

[22] P. Hande, S. Rangan, M. Chiang, and X. Wu, "Distributed uplink power control for optimal SIR assignment in cellular data networks," IEEE/ACM Trans. Netw., vol. 16, no. 6, pp. 1420-1433, Dec. 2008.

[23] M. Husso, Z. Zheng, J. Hamalainen, and E. Mutafungwa, "Dominant interferer mitigation in closed femtocell deployment," in Proc. 2010 IEEE Int. Symp. Personal, Indoor Mobile Radio Commun. Workshops, pp. $169-174$.

[24] S. Ryoo, C. Joo, and S. Bahk, "Spectrum allocation with beamforming antenna in heterogeneous overlaying networks," in Proc. 2010 IEEE Int. Symp. Personal, Indoor Mobile Radio Commun., pp. 1150-1155.

[25] S. Park, W. Seo, Y. Kim, S. Lim, and D. Hong, "Beam subset selection strategy for interference reduction in two-tier femtocell networks," IEEE Trans. Wireless Commun., vol. 9, no. 11, pp. 3440-3449, Nov. 2010.

[26] S. Park, W. Seo, S. Choi, and D. Hong, "A beamforming codebook restriction for cross-tier interference coordination in two-tier femtocell networks," IEEE Trans. Veh. Technol., vol. 60, no. 4, pp. 1651-1663, May 2011.

[27] S. D. Roy, S. Mondal, and S. Kundu, "Performance of joint admission and power control algorithms in cognitive-CDMA network," in Proc. 2010 Int. Conf. Comput. Commun. Netw. Technol., pp. 1-6.

[28] V. Chandrasekhar, J. G. Andrews, T. Muharemovic, and Z. Shen, "Power control in two-tier femtocell networks," IEEE Trans. Wireless Commun., vol. 8, no. 8, pp. 4316-4328, Aug. 2009.

[29] J. Zander, "Distributed cochannel interference control in cellular radio systems," IEEE Trans. Veh. Technol., vol. 41, no. 3, pp. 305-311, Aug. 1992.

[30] — , "Performance of optimum transmitter power control in cellular radio systems," IEEE Trans. Veh. Technol., vol. 41, no. 1, pp. 57-62, Feb. 1992.

[31] S. Koskie and Z. Gajic, "A Nash game algorithm for SIR-based power control in 3G wireless CDMA networks," IEEE/ACM Trans. Netw., vol. 13, no. 5, pp. 1017-1026, Oct. 2005.

[32] D. Fudenberg and J. Tirole, Game Theory. MIT Press, 1991.

[33] R. D. Yates, "A framework for uplink power control in cellular radio systems," IEEE J. Sel. Areas Commun., vol. 13, no. 7, pp. 1341-1347, Sep. 1995. 


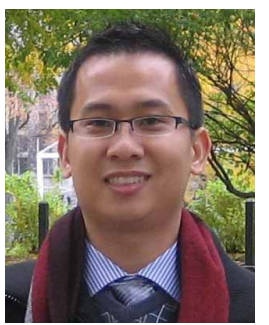

Duy Trong Ngo (Danny) (S'08) received the B.Eng. (with First-class Honours and the University Medal) degree in telecommunication engineering from the University of New South Wales, Sydney, NSW, Australia, in 2007, and the M.Sc. degree in electrical engineering (communication) from the University of Alberta, Edmonton, AB, Canada, in 2009. He is currently working toward the Ph.D. degree in electrical engineering with the Department of Electrical and Computer Engineering, McGill University, Montréal, QC, Canada.

His research interest is in the area of resource allocation for wireless communications systems with special emphasis on heterogeneous networks.

Mr. Ngo's undergraduate education was sponsored by the Federal Government of Australia through the Australian Development Scholarship scheme. He received the 2006 National Information and Communication Technology Australia (NICTA) Telecommunications Excellence Award. The highest standing telecommunication engineering graduate, he was awarded the University Medal by the University of New South Wales in 2007. From 2007 to 2009, he received the Alberta Ingenuity Foundation Student Scholarship and the Informatics Circles of Research Excellence ( $i$ CORE) Information and Communication Technology Graduate Student Award. He is currently the recipient of the Alexander Graham Bell Canada Graduate Scholarship from the Federal Government of Canada, as well as the McGill Engineering Doctoral Award.

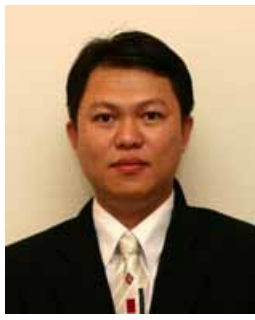

Long Bao Le (S'04-M'07) received the B.Eng. (with Highest Distinction) degree from Ho Chi Minh City University of Technology, Vietnam, in 1999 , the M.Eng. degree from Asian Institute of Technology, Pathumthani, Thailand, in 2002, and the Ph.D. degree from the University of Manitoba, Winnipeg, MB, Canada, in 2007.

From 2008 to 2010 , he was a postdoctoral research associate with Massachusetts Institute of Technology, Cambridge, MA. Since 2010, he has been an assistant professor with the Institut National de la Recherche Scientifique (INRS), Université du Québec, Montréal, QC Canada, where he leads a research group working on cognitive radio and dynamic spectrum sharing, radio resource management, network control and optimization.

Dr. Le is a member of the editorial board of IEEE WIRELESS COMMUNICATIONS LETTERS. He has served as the technical program committee co-chairs of the Wireless Networks track at IEEE VTC'2011-Fall and the Cognitive Radio and Spectrum Management track at IEEE PIMRC'2011.

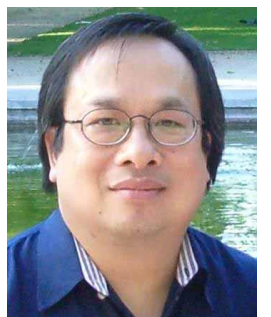

Tho Le-Ngoc (F'97) received the B.Eng. (with Distinction) degree in electrical engineering in 1976, the M.Eng. degree in microprocessor applications in 1978 from McGill University, Montréal, QC, Canada, and the Ph.D. degree in digital communications in 1983 from the University of Ottawa, Ottawa, ON, Canada.

From 1977 to 1982, he was with Spar Aerospace Limited, where he was involved in the development and design of satellite communications systems. From 1982 to 1985, he was an Engineering Manager of the Radio Group in the Department of Development Engineering of SRTelecom Inc., where he developed the new point-to-multipoint DA-TDMA/TDM Subscriber Radio System SR500. From 1985 to 2000, he was a Professor with the Department of Electrical and Computer Engineering, Concordia University, Montréal, QC, Canada. Since 2000, he has been a Professor with the Department of Electrical and Computer Engineering, McGill University, Montréal, QC, Canada. His research interest is in the area of broadband digital communications.

Dr. Le-Ngoc is a Senior Member of the Ordre des Ingénieurs du Québec and a Fellow of the Institute of Electrical and Electronics Engineers, the Engineering Institute of Canada, the Canadian Academy of Engineering, and the Royal Society of Canada. He is the recipient of the 2004 Canadian Award in Telecommunications Research, and the 2005 IEEE Canada Fessenden Award. He is the Canada Research Chair (Tier I) on Broadband Access Communications and the Bell Canada/NSERC Industrial Research Chair on Performance \& Resource Management in Broadband xDSL Access Networks.

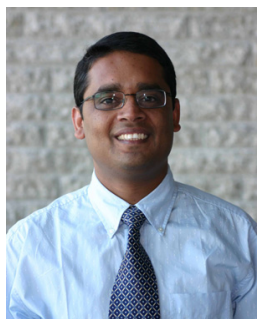

Ekram Hossain (S'98-M'01-SM'06) is a Professor in the Department of Electrical and Computer Engineering at University of Manitoba, Winnipeg, MB, Canada. He received his Ph.D. in electrical engineering from University of Victoria, Victoria, BC, Canada, in 2001. His current research interests include design, analysis, and optimization of wireless/mobile communications networks and cognitive radio systems (http://www.ee.umanitoba.ca/ ekram). Dr. Hossain served as the Area Editor for the IEEE TRANSACTIONS ON WIRELESS COMMUNICATIONS in the area of "Resource Management and Multiple Access" from 2009-2011. He is currently an Editor for the IEEE TRANSACTIONS ON MOBILE COMPUTING, IEEE WiRELESS COMMUNICATIONS, and the Editor-in-Chief for the IEEE COMMUNICATIONS SuRVEYS AND TUTORIALS (for the term 2012-2013). Dr. Hossain has several research awards to his credit which include the University of Manitoba Merit Award in 2010 (for Research and Scholarly Activities) and the 2011 IEEE Communications Society Fred Ellersick Prize Paper Award. $\mathrm{He}$ is a ComSoc Distinguished Lecturer for 2012 and 2013. Dr. Hossain is a registered Professional Engineer in the province of Manitoba, Canada.

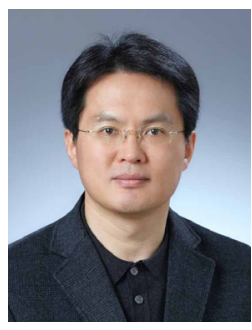

Dong In Kim (S'89-M'91-SM'02) received the B.S. and M.S. degrees in electronics engineering from Seoul National University, Seoul, Korea, in 1980 and 1984, respectively, and the M.S. and Ph.D. degrees in electrical engineering from University of Southern California (USC), Los Angeles, CA, in 1987 and 1990, respectively.

From 1984 to 1985 , he was a Researcher with Korea Telecom Research Center, Seoul. From 1986 to 1988 , he was a Korean Government Graduate Fellow in the Department of Electrical Engineering, USC. From 1991 to 2002, he was with the University of Seoul, Seoul, leading the Wireless Communications Research Group. From 2002 to 2007, he was a tenured Full Professor in the School of Engineering Science, Simon Fraser University, Burnaby, BC, Canada. From 1999 to 2000, he was a Visiting Professor at the University of Victoria, Victoria, BC. Since 2007, he has been with Sungkyunkwan University (SKKU), Suwon, Korea, where he is a Professor and SKKU Fellow in the School of Information and Communication Engineering. Since 1988, he is engaged in the research activities in the areas of wideband wireless transmission and access. His current research interests include cooperative relaying and base station cooperation, interference management for HetNet, cross-layer design and optimization.

Dr. Kim has served as an Editor and Area Editor for Cross-layer Design and Optimization for the IEEE TRANSACTIONS ON WIRELESS COMMUNICATIONS from 2002 to 2011, and also served as Co-Editor-in-Chief for the JOURNAL OF COMMUNICATIONS AND NETWORKS from 2008 to 2011. He is currently an Editor for Spread Spectrum Transmission and Access for the IEEE TRANSACTIONS ON COMMUNICATIONS and Founding Editor-in-Chief for the IEEE WIRELESS COMMUNICATIONS LETTERS. 